\title{
Dietary supplements and nutraceuticals for tinnitus
}

\begin{abstract}
Recommending an over-the-counter product for tinnitus should be done with caution. Each ingredient should be evaluated to determine of that ingredient might be potentially harmful to the patient. The U.S. Food and Drug Administration classify dietary as food.

None of the dietary supplements or nutraceuticals purporting relief from tinnitus has been approved by the US Food and Drug Administration. There are ingredients in some products that could have detrimental effects on special populations, especially cardiac patients.
\end{abstract}

Keywords: over-the-counter, dietary supplements, nutraceuticals, fda
Volume 9 Issue I - 2017

Robert M DiSogra
Osborne College of Audiology Salus University, USA

Correspondence: Robert M DiSogra Chairperson of the College Advisory Board Osborne College of Audiology Salus University Elkins Park Pennsylvania, USA,

Email bobdI030@aol.com

Received: April 19, 2017| Published: November 06, 2017
Abbreviations: OTC, over-the-counter; FDA, food and drug administration; DSHEA, dietary supplement health and education

\section{Introduction}

Physicians and audiologists encounter patient's everyday that has tinnitus. In most cases it is cochlear in origin however persons with tinnitus - in the absence of hearing loss - need to be further evaluated.

Identifying an organic origin will help to further manage the patient but could the tinnitus be an adverse side effect of a particular pharmaceutical they are taking for another medical condition? A comprehensive list of pharmaceuticals with auditory/perceptual side effects can be referenced on line at www.earservsecure.com/drugs. Other websites include www.rxlist.com or www.drugs.com [not an endorsement.

Although tinnitus may have been a reported side effect during a drug's clinical trials (prior to FDA approval) the incidence might be extremely low. Therefore it is critical to establish a time line from when the drug was first introduced and the tinnitus was first noticed. However, a patient who self medicates to manage their tinnitus with an over-the-counter (OTC) product (without consulting an otologist or audiologist) can be just a few internet clicks away from trouble.

\section{Who's minding the store on OTC tinnitus products?}

The United States Food and Drug Administration (FDA) (www. fda.gov) along with the Federal Trade Commission (FTC) (www.ftc. gov) are responsible for seeing that nutritional supplements are safe. There is also a federal Office of Dietary Supplements at the National Institutes of Health (http://ods.od.nih.gov) and the United States Pharmacopoeia (www.usp.org).

All vitamin/herbal supplement manufacturers must register their product with the FDA before being offered for sale to the public. Having a product registered with the FDA does not imply approval by the FDA. Several products state that their product is "FDA approved" -actually, it is approved for sale and not because of any evidence based research that has established efficacy and safety.

\section{Over-the-counter tinnitus relief products}

The current research by the author has identified over 80 products (herbal supplements, vitamins, homeopathic remedies, essential oils, laser lights and mechanical devices) all claiming to either temporarily or permanently relieve tinnitus. These products are available without a prescription and may contain some ingredients that might be harmful to cardiac patients, pregnant or nursing mothers or children. Some ingredients also appear on the FDA's poisonous plants list. ${ }^{1}$

Herbals (Roots, Stems and Leaves), traditional Chinese medicine and other non-scientifically evaluated ingredients are mixed into pills, liquids, sprays or powders with dosages usually reported in milligrams or micrograms. On the label it is not unusual to see asterisks indicating that no minimum daily value has been established. ${ }^{2}$

Claims about safety and efficacy do not have to be proven because the U.S. Food and Drug Administration classifies herbals/nutritional supplements as food. Consequently, the manufacturer does not have to go through all the scientific scrutiny that a pharmaceutical must go through prior to FDA approval. All the product's manufacturer needs to do is print the following statement on their labels: These statements have not been evaluated by the Food and Drug Administration. This product is not intended to diagnose, treat or cure any disease."

Patients can pay anywhere from a few dollars to over US $\$ 50.00$ per bottle for a one month supply (usually with a three month minimum supply recommended) with no scientifically proven guarantee for relief. ${ }^{3,4}$

Therefore, when your patient asks for advice on OTC tinnitus relief products (they usually will bring in an ad from a magazine or newspaper) on the latest 'cure' for tinnitus, it is the author's intention that you caution them that none of these products are approved or endorsed by the Food and Drug Administration and that they are not intended to diagnose, treat or cure any disease.

\section{Published research - does it exist?}

Peer reviewed research on $90 \%$ of the combined ingredients does not exist. Information that appears on commercial websites usually has no citations to support the claims made by the manufacturer or distributor. It is not unusual to read comments such as "helps reduce ear noises." or "studies have shown that XYZ can reduce ringing noises in the ear." The challenge is to track down those studies which, for the most part, were not in any peer-reviewed journal. Therefore, double-blind, clinical studies using acceptable research methods will be lacking.

\section{Use of the DSHEA disclaimer}

Under the Dietary Supplement Health and Education Act of 1994 (DSHEA), the dietary supplement or dietary ingredient manufacturer 
is responsible for ensuring that a dietary supplement or ingredient is safe before it is marketed. FDA is responsible for taking action against any unsafe dietary supplement product after it reaches the market. However, a disclaimer can be used freeing the manufacturer of providing efficacy and safety data from recognized clinical trials. The label statement reads: "These statements have not been evaluated by the FDA. This product is not intended to diagnose, treat, cure or prevent any disease."

\section{Combined ingredients for tinnitus relief}

There is little scientific research about the effectiveness of the combination of the ingredients found in OTC tinnitus relief products that can support its use in a tinnitus management program..$^{5-8}$

Enrico. ${ }^{7}$ concluded that "...the use of CAM (Complimentary Alternative Medicine) products in tinnitus therapy in general lack substantial scientific support, and that these substances are probably not clinically effective either. However, it is difficult to draw clear-cut conclusions regarding CAM pharmacological approach to tinnitus. In fact, the subjective nature of tinnitus and the reported variability in patient's response to therapy indicate that several non-pharmacological factors may be influencing drug effects, with the placebo effect playing a major role. Nevertheless, in view of the potential harm that may occur from inappropriate use of CAM products, physicians need to be aware of their principal characteristics with particular emphasis on toxicity and possibilities of interaction with prescription drugs."

\section{Conflicts of interest}

Author declares there are no conflicts of interest.

\section{Acknowledgments}

None.

\section{Funding}

None.

\section{References}

1. Disogra RM. Guide to over-the-counter tinnitus relief products. (2nd edn), Oaktree Products. 2006.

2. Disogra RM. Adverse herbal \& nutritional/dietary supplement side effects. Audiology Today. 2012;24(6):40.

3. Disogra RM. Alpha Lipoic Acid and Tinnitus. 2010.

4. Disogra RM. Beta-carotene and tinnitus. 2007.

5. Disogra RM. Pharmacology and ototoxicity for audiologist. Campbell, K.C.M Demler. 2006.

6. Disogra RM. Adverse herbal medicine reactions and audiology practice. Audiology Today. 2003.

7. Enrico P, Sirca D, Mereu M. Antioxidants, minerals, vitamins, and herbal remedies in tinnitus therapy. Prog Brain Res. 2007;166:323-330.

8. Disogra RM Adverse drug reactions. 